\title{
Tracción de canino maxilar con la técnica quirúrgica incisión vertical y túnel de acceso subperióstico
}

\section{Maxillary canine traction with surgery technique vertical incision and subperiosteal tunnel access}

\author{
Eilien Tovío Martínez ${ }^{1}$, Patricia Vergara Villarreal ${ }^{2}{ }^{(-)}$, Adel Martínez Martínez ${ }^{3}{ }^{(0)}$, Jonathan \\ Harris-Ricardo 4 (iD
}

Tipología: Reporte de caso clínico.

Para citar este artículo: Tovío Martínez E, Vergara Villarreal P, Martínez Martínez A, Harris-Ricardo J. Tracción de canino maxilar con la técnica quirúrgica incisión vertical y túnel de acceso subperióstico. Duazary. 2019 septiembre; 16(3): 104 - 111. Doi: http://dx.doi.org/10.21676/2389783X.2973

Recibido en junio 25 de 2018

Aceptado en marzo 15 de 2019

Publicado en línea en septiembre 01 de 2019

\section{RESUMEN}

Los dientes caninos presentan una alta frecuencia de inclusión, ocupando el segundo lugar después de los terceros molares. Su permanencia en la arcada dental es importante por las diversas funciones que cumplen en la cavidad bucal: permiten desgarrar los alimentos al masticar, guían la oclusión dental, son los encargados de centralizar y desprogramar la oclusión durante los movimientos de lateralidad, dan soporte al labio superior y marcan el límite del sector anterior, destacándose en la sonrisa. La técnica de incisión vertical y túnel de acceso subperióstico (VISTA) permite la tracción de dientes impactados por vestibular, es poco traumática para los tejidos blandos, disminuye los problemas periodontales y evita la reabsorción radicular de los dientes adyacentes. El objetivo es reportar un caso clínico en el que se especifica la secuencia del abordaje ortoquirúrgico aplicando la técnica VISTA en un paciente con el diente 13 incluido, el cual mostró buenos resultados. Se describen la técnica quirúrgica y manejo ortodóntico.

Palabras clave: diente canino; maxilar; ortodoncia; resorción radicular.

1. Universidad de Cartagena. Cartagena, Colombia. Correo: etoviom@ unicartagena.edu.co - https://orcid.org/0000-0003-3702-2791.

2. Universidad de Cartagena. Cartagena, Colombia. Correo: Vergara_patri@ hotmail.com - https://orcid.org/0000-0002-5794-290X

3. Universidad de Cartagena. Cartagena, Colombia. Correo: amartinezm4@ unicartagena.edu.co - https://orcid.org/0000-0001-7008-2222

4. Corporación Universitaria Rafael Núñez. Cartagena, Colombia. Correo: j.harris.r@ hotmail.com - https://orcid.org/0000-0002-46620729 


\section{ABSTRACT}

The canine teeth present a high frequency of inclusion, occupying the second place after the third molars, its permanence in the dental arcade is important for the various functions that it fulfills in the oral cavity, allowing tearing of foods by chewing, guiding dental occlusion, are responsible for centralizing and deprogram the occlusion during lateral movements, support the upper lip, mark the limit of the anterior sector, standing out in the smile, the technique vertical incision and subperiosteal tunnel access (VISTA) Allows the traction of teeth impacted by vestibular, it is not very traumatic for the soft tissues, it diminishes the periodontal problems and it avoids the root resorption of the adjacent teeth. The objective is to report a clinical case in which the sequence of the ortho-surgical approach is specified by applying the VISTA technique to a patient with the included tooth 13, showing good results, describing the surgical technique and orthodontic management.

Keywords: cuspid; maxilla; orthodontics; root resorption.

\section{INTRODUCCIÓN}

Los caninos son dientes que ocupan un lugar importante en el esquema de oclusión y que permiten desgarrar los alimentos en el momento de la masticación, al tiempo que ayudan en el buen contorno de la cara y a conformar una estética aceptable de los dientes ${ }^{1}$. El tamaño y la longitud de su raíz son vitales en la continuidad de los arcos dentarios, siendo responsables del funcionamiento y la armonía oclusal' ${ }^{2}$. Asimismo, desde el punto de vista funcional, el canino guía el desplazamiento en lateralidad, coordina la función neuromuscular gracias a los impulsos propioceptivos periodontales, conduce el movimiento de cierre mandibular en relación céntrica y ayuda a reducir los traumatismos a nivel de la articulación temporomandibular. Por todos estos motivos, la permanencia de estos dientes en la boca es esencial para el sistema estomatognático ${ }^{3}$.

Según la frecuencia de inclusión de los diversos dientes, el canino superior ocupa el segundo lugar después del tercer molar mandibular ${ }^{4}$. Las opciones de tratamiento van a depender del tipo de retención, la severidad de la impactación, la posición del diente, la edad, entre otros ${ }^{5}$. Es habitual que el manejo de la terapéutica sea ortoquirúrgico, con la finalidad de posicionar adecuadamente el diente en la arcada. Es así como se observa que la mayoría de casos requiere de una intervención quirúrgica para remoción, exposición o trasplante, con o sin la tracción ortodóntica ${ }^{6}$.
Entre los tratamientos para inclusión se destaca la técnica de incisión vertical y túnel de acceso subperióstico (VISTA), creada en principio por el doctor Homayoun Zadeh para resolver problemas periodontales ${ }^{7}$, y posteriormente modificada por Chris Chang para la tracción de dientes superiores impactados por vestibular ${ }^{8}$. Este es un procedimiento poco traumático para los tejidos blandos que disminuye los problemas periodontales y evita la reabsorción radicular de los dientes adyacentes. El objetivo es posicionar el diente que se tracciona en la arcada dental utilizando elementos como minitornillos, cadenas elásticas y botones ortodónticos ${ }^{9}$.

El uso de minitornillos ofrece ventajas comparativas frente a métodos tradicionales de anclaje ortodóntico, proporcionando un anclaje óseo estable durante el tratamiento ${ }^{10}$. En este caso, el área infracigomática es la que se utiliza para el anclaje absoluto del minitornillo porque aporta una gran porción ósea para la retención. Su colocación no debe sobrepasar los $15 \mathrm{~mm}$ de profundidad desde el borde inferior del proceso alveolar $^{11}$.

El presente reporte tiene como propósito mostrar la secuencia clínica del abordaje quirúrgico para tracción del canino incluido mediante la técnica VISTA. 


\section{REPORTE DE CASO}

Paciente femenina de 11 años que fue remitida por ortodoncia al servicio de estomatología y cirugía oral de la clínica odontológica de la Universidad de Cartagena por presentar diente canino incluido. En los antecedentes y revisión por sistemas reporta no presentar alteraciones.

En el examen físico general se encontró el tercio facial inferior aumentado, con escalón labial ligeramente negativo e hipertonía del mentón. En la exploración intraoral se observó relación molar bilateral de clase II, con ausencia de espacio para la erupción de caninos superiores, apiñamiento anteroinferior severo y arco superior triangular y estrecho, característico de un paciente respirador oral.

En el estudio radiográfico, la ortopantomografía muestra impactación moderada del diente 13 en posición vestibular, cuya porción coronal esta próxima al tercio apical de los dientes 11 y 12 (Figura 1). En el análisis cefalométrico de Steiner, los ángulos de evaluación antero posterior se encuentran alterados SNA: $77^{\circ}$, SNB $70^{\circ}$, ANB $5^{\circ}$, GoGn.SN: $43^{\circ}$, Ocl.SN: $29^{\circ}$, lo que da como resultado una clase II esquelética, indicando retrognatismo mandibular y patrón de crecimiento vertical.

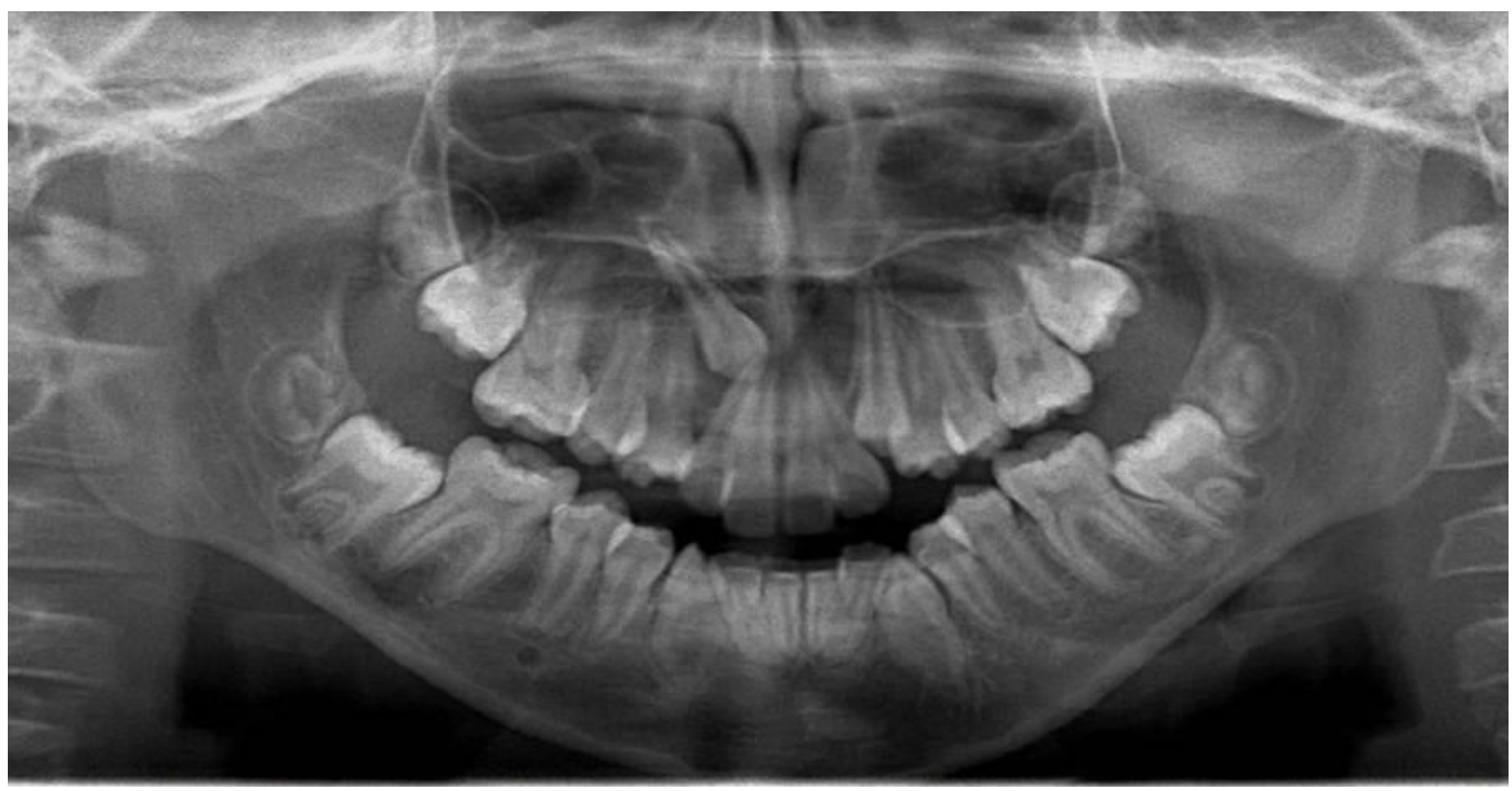

Figura 1. Impactación del diente 13 con inclinación mesial, en posición vestibular y próximo al tercio apical de los dientes 11 y 12

En junta multidisciplinar, incluyendo cirujano oral y ortodoncista, se evaluaron los hallazgos clínicos y radiográficos con el fin de elegir el tratamiento más acertado para la paciente. Como resultado se decidió llevar a cabo la técnica VISTA con fines ortodónticos para traccionar el diente 13, colocarlo en la posición correcta de la arcada dental y evitar la reabsorción radicular de los dientes adyacentes.

\section{Tratamiento quirúrgico}

Previamente, se les explicó el procedimiento a la paciente y a su madre, quien aceptó el tratamiento y autorizó a proceder, para lo cual se firmó el asentimiento y consentimiento informado. La intervención quirúrgica, hecha bajo anestesia local, empezó con una incisión vertical en distal de la zona del diente 13, involucrando la encía libre hasta la línea 
mucogingival, sin comprometer la encía adherida y la mucosa de revestimiento. Luego se desprendió el tejido blando, sindesmotomía y osteotomía, para exponer la corona del diente (Figura 2A). Posteriormente se procedió a la cementación de un botón en la cara vestibular y se continuó con osteotomía conductiva, que implicó una decorticacion distal al órgano 13. De este modo se creó un canal o ruta de tracción del diente que permitió mayor movimiento de la estructura dental impactada. Enseguida se creó un túnel subperióstico desde la zona del diente 13 hasta la zona del diente 16 y se practicó disección del tejido blando. Después se colocó un minitornillo infracigomático en zona de dientes 16 y 17 para proporcionar anclaje y dirigir los movimientos aplicando fuerzas de 60 grados con el fin de distalizar el diente impactado (Figura 2B) y colocarlo en la zona ideal para la erupción. Acto seguido, se hizo una incisión vertical de $3 \mathrm{~mm}$ en la zona del diente 16 y se pasó una cadena elástica de segunda generación por el túnel subperióstico, que iba del botón de tracción en el diente 13 hasta el minitornillo infracigomático (Figura 3). Finalmente, se procedió a hemostasia y sutura. En la misma intervención quirúrgica se hicieron las exodoncias de los dientes 14 y 24 debido a la falta de espacio para la erupción de 13 y 23.

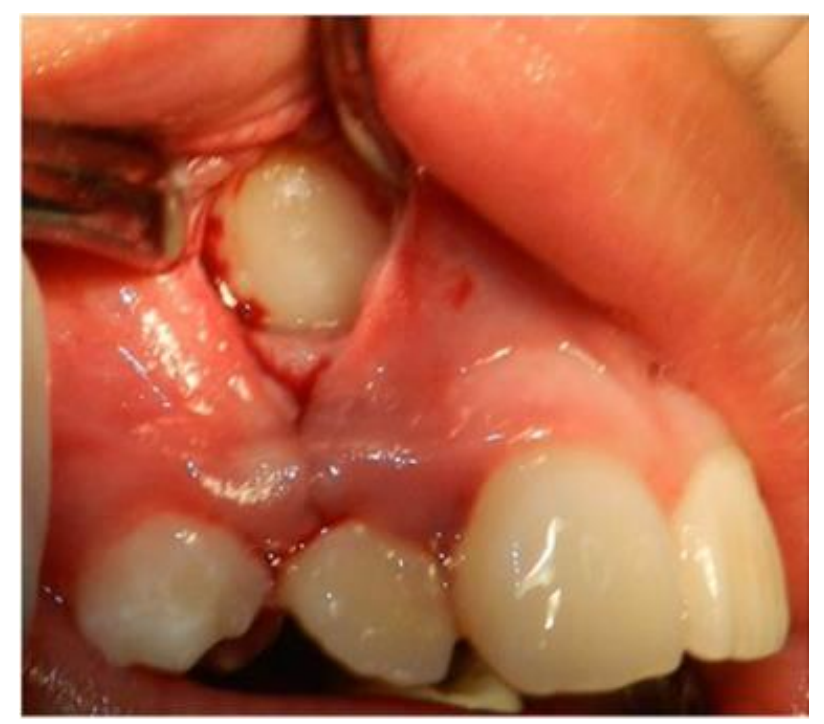

Figura 2A. Exposición coronal del diente impactado

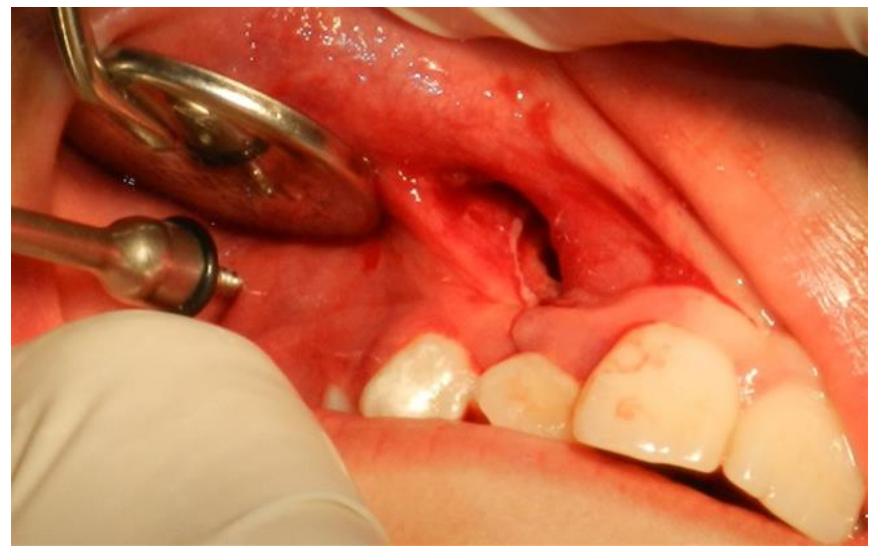

Figura 2B. Minitornillo infracigomático en zona de dientes 16 y 17 


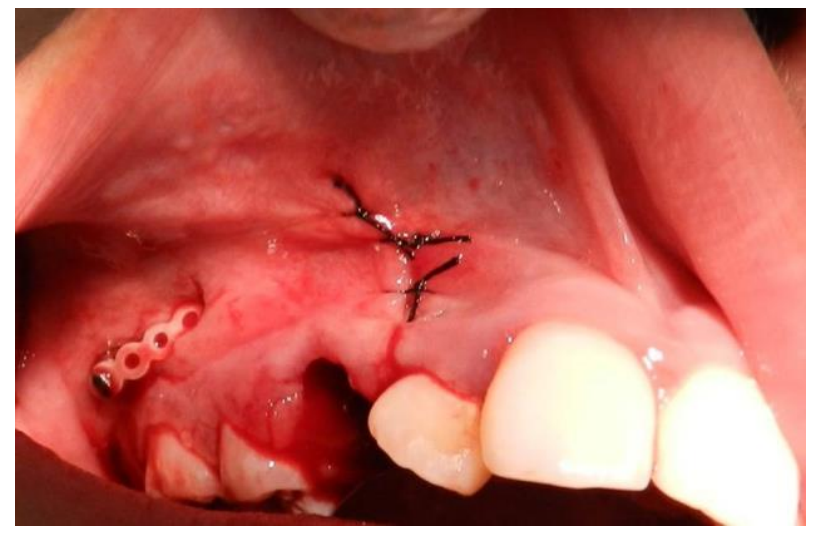

Figura 3. Paso de la cadena elástica por el túnel subperióstico, la cual va desde el botón de tracción en el diente 13 hasta el minitorinillo infracigomático

En el control posoperatorio a los ocho días se evidenciaron tejidos blandos en buen proceso de cicatrización. Se inició la cementación de los brackets de autoligado, menos en el diente 12, para impedir que con el movimiento ortodóntico los dientes contacten, evitando reabsorción radicular. La secuencia de arcos que se siguió fue determinada por las condiciones de alineación de la arcada superior, iniciando con un alambre nitinol termoactivado 0,014-0,016-0,16 x 0,22, y se activó la cadeneta a razón de un eslabón cada 15 días.
Posteriormente, a los dos meses y medio, se realizó control radiográfico con ortopantomografía, en el que se observó retracción y leve distalización del diente 13, localizado en el tercio medio radicular del diente 12 y en posición mesioinclinado. Se destaca que inicialmente se encontraba a nivel apical de los dientes 11 y 12 , de manera que se evidenció mejoría en la posición. Se continuaron las activaciones, y en el control radiográfico del cuarto mes el diente 13 se encontró en posición vertical, con adecuado espacio para la erupción (Figura 4).

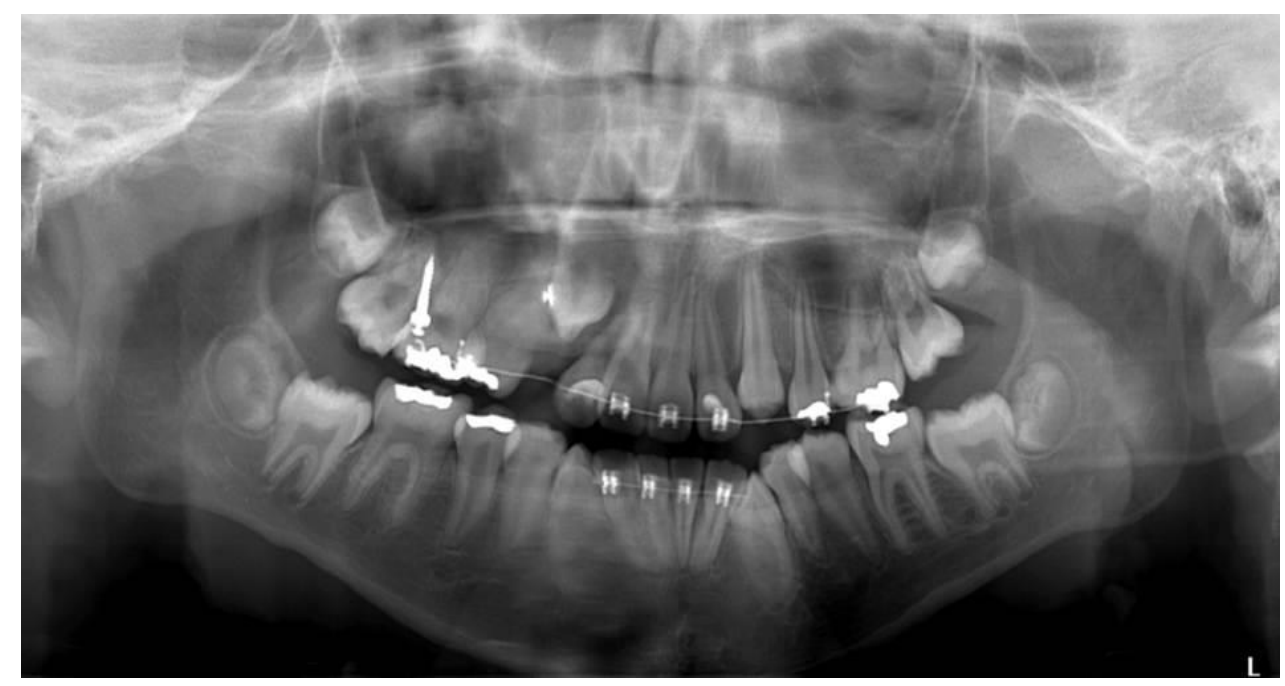

Figura 4. Control radiográfico al cuarto mes en el que se observó diente 13 en posición vertical, con adecuado espacio para la erupción 
En el control clínico del mes seis se observó exposición completa de la corona del diente 13, descendiendo en posición vertical, siendo esta la posición adecuada en la arcada dental. Faltaban pocos milímetros para descender hasta el plano oclusal (Figura 5A-5B).

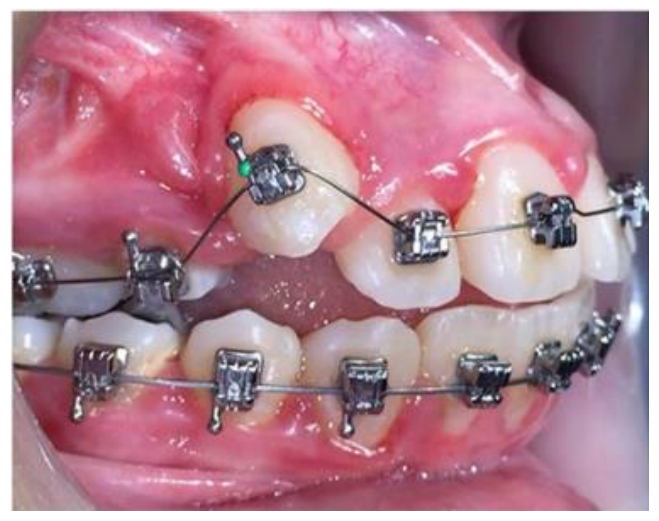

Figura 5A. Vista anterior del control clínico al mes seis, donde se observó exposición completa de la corona del diente 13 , descendiendo en posición vertical

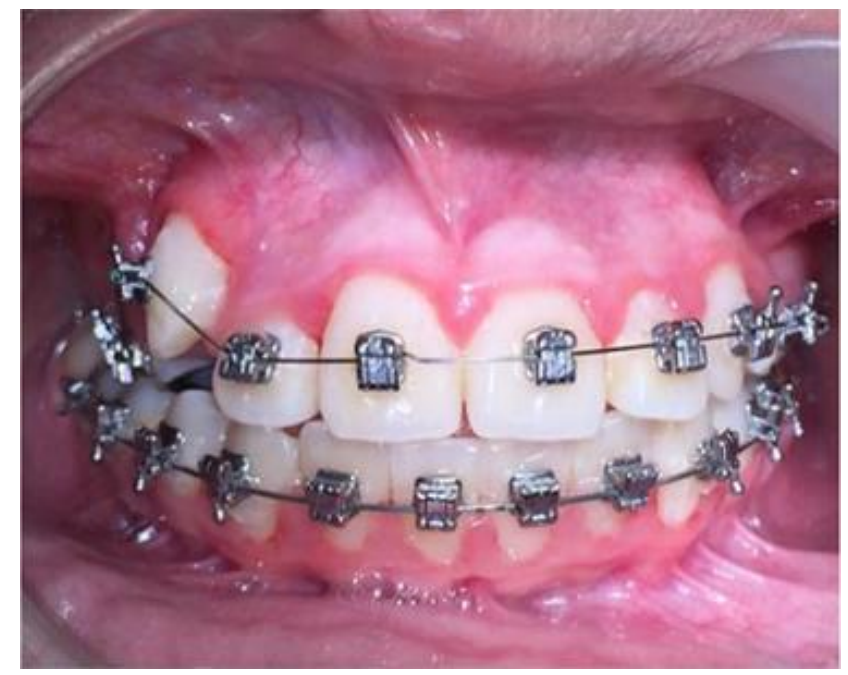

Figura 5B. Vista lateral del control clínico al mes seis, donde se observó diente 13 en la posición adecuada en la arcada dental, faltando pocos milímetros para descender hasta el plano oclusal

\section{Declaración sobre aspectos éticos}

En la realización y participación del presente estudio se tuvo en cuenta la firma del asentimiento y consentimiento informado por parte de la paciente y sus padres. Además, los procedimientos seguidos se conformaron a las normas éticas de la Resolución 8430 de 1993 del Ministerio de Salud y Protección Social de Colombia y a la Declaración de Helsinki.

\section{DISCUSIÓN}

Al canino superior permanente se le atribuye suma importancia en la arcada dental por su lugar en el esquema de la oclusión funcional, además de su contribución a la estética del paciente. En ese mismo sentido se destacan su tamaño, la longitud de raíz y el papel que 
desarrolla en la estabilidad de los arcos dentarios $^{12,13}$.

Mittal et al. ${ }^{14}$ afirman que la inclusión del diente canino está fuertemente asociada con la falta de espacio o el apiñamiento del arco alveolar, discrepancias del arco dentario maxilar, posiciones anormales, anquilosis, trauma o alteraciones patológicas como quistes, entre otras, así como con el desplazamiento del folículo dentario, el aumento de la presión intravascular dentro del germen dentario y procesos infecciosos crónicos. Estos hallazgos coincidien con el presente reporte de una paciente femenina con impactación moderada del diente 13, en posición vestibular, con la porción coronal próxima al tercio apical de los dientes 11 y 12, la cual le ocasionó alteraciones como mal oclusión, rotación dental, apiñamiento, reabsorción radicular y migración del diente vecino.

Con relación a la técnica quirúrgica VISTA con fines ortodónticos, $\mathrm{Su}$ et al. ${ }^{8}$ reportaron un caso de un paciente que presentó el diente 23 impactado por vestibular en el que aplicaron la técnica VISTA para la tracción dental, con un anclaje aportado por minitornillo infracigomático para proporcionar fuerza de tracción, sin producir movimiento no deseado de otros dientes. Este tratamiento arrojó buenos resultados después de seis meses, lo cual concuerda con el actual reporte en el que se implementó la técnica quirúrgica VISTA con minitornillo infracigomático, para la tracción del diente 13 impactado por vestibular, impidiendo que con el movimiento ortodóntico los dientes contacten y evitando reabsorción radicular. De esta forma se pudo ubicar el canino en una posición que le permitió el descenso vertical para la erupción y el lugar correspondiente en la arcada dental después del mismo lapso.

El abordaje ortoquirúrgico brinda una solución efectiva a los problemas de inclusión dentaria al restituir la función y la estética deseadas del sistema estomatognático ${ }^{15}$. Autores como $\mathrm{Su}$ et $a l{ }^{8}$ afirman que la técnica VISTA para la tracción dental es una alternativa de tratamiento poco traumática que disminuye los problemas periodontales y evita la reabsorción radicular de los dientes adyacentes, ventajas que se presentaron en los resultados obtenidos en el actual reporte.

\section{CONCLUSIÓN}

La técnica quirúrgica de incisión vertical y túnel de acceso subperióstico es una alternativa terapéutica ortoquirúrgico que mostró resultados favorables relacionados con la tracción de canino maxilar y evitó la reabsorción radicular de los dientes adyacentes. Mediante ella se consiguió distalizar el diente incluido y posicionarlo adecuadamente en la arcada dental en seis meses de tratamiento.

\section{DECLARACIÓN SOBRE CONFLICTOS DE INTERESES}

Los autores declaran no presentar conflicto de interés.

\section{CONTRIBUCIÓN DE LOS AUTORES}

Primer autor: elaboración del manuscrito y redacción.

Segundo autor: trabajo de campo y aporte de la temática.

Tercer autor: trabajo de campo y aporte de la temática.

Cuarto autor: elaboración del manuscrito y redacción.

\section{REFERENCIAS BIBLIOGRÁFICAS}

1. Park JH, Srisurapol T, Tai K. Impacted maxillary canines: diagnosis and management. Dent Today. 2012 Sep;31(9):62-64. 
2. Thirunavukkarasu R, Sriram G, Satish R. The orthodontic management of ectopic canine. J Pharm Bioallied Sci. 2015;7(Suppl 2):749-751.

3. Madsen DP. The impacted canine--an orthodontic perspective. Ann R Australas Coll Dent Surg. 2012;21:111-2.

4. Counihan K, Al-Awadhi EA, Butler J. Guidelines for the assessment of the impacted maxillary canine. Dent Update. 2013;40 (9):7702, 775-7.

5. Bedoya MM, Park JH. A review of the diagnosis and management of impacted maxillary canines. J Am Dent Assoc. 2009;140(12):1485-93. Doi: https://doi.org/10.14219/jada.archive.2009.009 9

6. Montenegro M, Hara F. Tracción de canino retenido superior con aparatología ortodóntica fija Edgewise: Reporte de un caso. Revista Mexicana de Ortodoncia. 2013;1(1):62-72. Doi: https://doi.org/10.1016/S2395-9215(16)300095

7. Zadeh HH. Minimally invasive treatment of maxillary anterior gingival recession defects by vestibular incision subperiosteal tunnel access and platelet-derived growth factor BB. Int $\mathbf{J}$ Periodontics Restorative Dent. 2011;31(6):653660

8. Su B, Chang $\mathrm{CH}$, Roberts WE. Highly Positioned and Transalveolar Impacted Maxillary Canine. Int $\mathbf{J}$ Orthod Implantol. 2011;24(1):14-26.

9. Hsu YL, Chang CH, Roberts WE. Soft tissue considerations for the management of impactions. IJOI. 2011;24(1):50-59.

10. Bationo R, Jordana F, Kabore WA, Ngom PI. Two clinical examples of the use of orthodontic mini-screws. Odontostomatol
11. López DF, Herrera-Guardiola S. Corrección de Maloclusión dental Clase II unilateral con Dispositivo de Anclaje Temporal Infracigomático. Ces Odont. 2015;28(2):142155.

12. Sukh R, Singh GP, Tandon P. Interdisciplinary approach for the management of bilaterally impacted maxillary canines. Contemp Clin Dent. 2014 Oct;5(4):539-44.

13. Troya BE, Martínez AJ, Padilla SE, Matos CMR. Tratamiento quirúrgico de caninos retenidos en el municipio Colón. Años 20132014. Rev Med Electrón. 2016;38(2):123-131.

14. Mittal M, Murray A, Sandler J. Impacted maxillary canines--a perennial problem. Dent Update. 2012;39(7):487-8, 491-2, 495-7. Doi: https://doi.org/10.12968/denu.2012.39.7.487

15. Becker A, Chaushu S. Surgical treatment of impacted canines: what the orthodontist would like the surgeon to know. Oral and Maxillofacial Surgery Clinics of North America. 2015;27(3):449-458.

Doi: https://doi.org/10.1016/j.coms.2015.04.00 7

Trop. 2016;39(153):5-13. 